\title{
The Hypopharynx of Male and Female Mosquitoes ${ }^{\S}$
}

\author{
Isra Wahid, ${ }^{*}$, Toshihiko Sunahara ${ }^{2}$ and Motoyoshi Mogi² \\ ${ }^{I}$ Department of Parasitology, Hasanuddin University School of Medicine, Jl. Perintis Kemerdekaan KM. 11 Tamalanrea, \\ Makassar 90245, Indonesia \\ ${ }^{2}$ Department of Parasitology, Saga University School of Medicine, Nabeshima 5-1-1, Saga Shi, Saga Ken, Japan
}

\begin{abstract}
In blood-feeder female mosquitoes, the hypopharynx stylet is one part of the fascicle, the structure that pierces into the host skin during blood feeding. As other parts, the hypopharynx is a free stylet. However, since male mosquitoes do not feed blood, their mouthparts are less developed. The hypopharynx fuses with the inner wall of the labium, while maxillae and mandibles are much shorter than the labium. Only the labrum and the labium are well developed and function as food canal and its sheath, respectively. Light microscopy (LM) and scanning electron microscopy (SEM) were done to compare the hypopharynx of males of several mosquito genera and, in addition, females of autogenous mosquitoes.

The hypopharynxs of males of both autogenous and anautogenous mosquitoes fuse with the labium inner wall as long as the labium length, but are distinctly different structures from the labium. Dissociation occurs on the hypopharynx of female autogenous mosquitoes: Toxorhynchites spp. have a free hypopharynx as in anautogeny mosquitoes, whereas it fuses with the labium wall in Malaya genurostris Leicester, as in male mosquitoes.
\end{abstract}

\section{INTRODUCTION}

As most of female mosquitoes feed on blood, their mouthparts are highly specialized for piercing the host skin and sucking blood. During blood feeding, the fascicle (contains a labrum, a hypopharynx, pairs of mandibles and maxillae) enters the host skin, while the labium-formed sheath remains outside the skin. The maxilla and the mandible function as piercing organs $[1,2]$, but there is no active movement of the hypopharynx. No muscles are either originated from or inserted on the hypopharynx [2]. Thus, practically, there is no active movement of the hypopharynx. Its function is to transfers saliva to the host tissue through salivary canal on its dorsal surface.

Unlike females, male mosquitoes do not feed on blood. Vizzi described the hypopharynx in Anopheles quadrimaculatus Say males as a sclerotic plate on the labial gutter [3], while Snodgrass stated that the hypopharynx and the labium of male mosquitoes completely fuse together forming the labio-hypopharynx [4]. Christophers recognized the hypopharynx of Aedes aegypti (Linneaus) males as a ridge on the labium [5]. Though the hypopharynx of Ae. aegypti males is not free from the labium wall, it is discernible from the labium wall by its texture both under light and scanning electron microscopy (SEM). Fusion of the hypopharynx with the labium is understandable, because the hypopharynx of males does not need to enter into the skin.

*Address correspondence to this author at the Department of Parasitology, Hasanuddin University School of Medicine, Jl. Perintis Kemerdekaan KM. 11 Tamalanrea, Makassar 90245, Indonesia; Tel: +62-411-580687; Fax: +62-411-586297; E-mail: israwahid@gmail.com

${ }^{\S}$ Note: Some parts of this work were presented in The $2^{\text {nd }}$ ASEAN Congress of Tropical Medicine and Parasitology, Bandung, Indonesia, May 21-23, 2006).

\section{MATERIALS AND METHODS}

\section{Mosquitoes}

Materials used were the same with those used for examination maxillae and mandibles of male mosquitoes in our previous study [1] listed in Table $\mathbf{1}$. The hypopharynx states of males of 44 species of 12 genera, females of 2 partially autogenous and 5 autogenous species were examined. For comparison, the hypopharynx of female Ae. aegypti was examined as a representative of anautogenous species. Specimens were kept in $70 \%$ ethanol solution, except for a few specimens kept in 3\% glutaraldehide solution for transmission electron microscopy (TEM).

\section{Light Microscopy}

Males of all the 44 species were examined under light microscopy. The specimens were stained with Fuschin Acid as desribed in Wahid et al. [6]. They then put in methyl cellosolve (Nakarai Chemicals, Kyoto, Japan) on a slide glass and dissected under a dissecting microscope. The head was separated from the thorax. The free hypopharynx of females, together with other free stylets, was easily pried up from the labium gutter by a fine insect pin. On the other hand, it was no easy to separate the hypopharynx of males from labium tissue. The clypeus was pulled upward and forward carefully, to separate the hypopharynx from the inner wall of the labium. The detached hypopharynx was then cover with cover glass and examined under a light microscopy.

\section{Scanning Electron Microscopy (SEM)}

SEM was used to confirm the observation made under light microscopy. For this purpose, only males of Ae. aegypti were examined, since all male specimens principally have the same attribute. The dissected head, taking the labrum, maxillae and mandibles out of the labial gutter and leaving the hypopharynx undisturbed, was put on a poly L lysinecoated glass slide. The specimens were gradually dehydrated 
through $70 \%$ to $99 \%$ ethanol, and then placed in $99 \% t$-butyl alcohol and kept in a freezer $\left(-20^{\circ} \mathrm{C}\right)$. They were freeze-dried by using an ID-2 drier (EIKO, Tokyo, Japan) prior to gold coating by an EIKO IB-3 ion coater, then examined by a JSM-5200 LV scanning electron microscope (JEOL, Tokyo, Japan).

\section{Transmission Electron Microscopy (TEM)}

Transversal section of the proboscis of males and females of Ae. aegypti, Malaya genurostris Leicester, Topomyia vijayae Ramalingan and Toxorhynchites splendes (Weidemann) were examined by TEM. Fresh or dry specimens were kept in 3\% glutaraldehyde solution (dry specimens were first put in $70 \%$ ethanol for \pm 5 seconds to make them sinkable completely into $3 \%$ glutaraldehyde). Heads were then separated from the rest of the body in 3\% glutaraldehyde solution buffered to $\mathrm{pH} 7.4$ with phosphate buffer, then fixed for at least $1 \mathrm{hr}$ in phosphate-buffered $1 \%$ osmium tetroxide solution. Dehydration was carried out by passing the head through $50 \%, 75 \%, 85 \%, 95 \%, 100 \%$ ethanol, each for 15 min, then, head was kept in n-glycildiethyl solution (QY-1) for 20-40 min, to a 1: 1 mixture of Epon and QY-1 for $1 \mathrm{hr}$, in a 3: 1 mixture of Epon-QY-1 overnight, and was embedded in Epon 812 for $2 \mathrm{~h}$ before heating at $45^{\circ} \mathrm{C}$ and $60^{\circ} \mathrm{C}$, each for $24 \mathrm{~h}$. The block was then cut with an ultra microtome. Sections were mounted on parlodion-carbon coated grids and stained in a saturated uranyl acetate solution followed by lead citrate. Specimens were examined by a transmission electron microscope JEM-1210 (JEOL, Tokyo, Japan).

\section{RESULTS}

\section{Hypopharynx of Males}

The hypopharynx of male mosquitoes invariably fuses with the inner wall of the labium for all the 44 species (Table 1). It is a thick longitudinal plate ventrally fused with the inner wall of the labium. Its proximal and distal ends connect to the cibarial ventral wall and the hairy ligula at the proboscis tip, respectively (Fig. 1). Contrasting to the longitudinally wrinkle surface of inner wall of the labium, the hypopharynx has a smooth surface and a gutter-like salivary canal at the median line (Fig. 2).

By light microscopy, the hypopharynx of males can be recognized as a longitudinal structure in the labial gutter with parallel edges and a midrib representing a salivary canal. A hypopharynx that is detached from the labium has two parallel edges at both sides. The inner edge is the hypopharynx edge and the outer one is the labium wall tissue that is attached to the hypopharynx (Fig. 3). The transversal section of the proboscis by transmission electron microscopy (TEM) shows different electron density between the hypopharynx tissue and the labium tissue (Fig. 4).

Anatomically, the salivary canal is a dorsally open gutter on the hypopharynx surface. However, its overlapping edges make it functionally a closed tube (Fig. 4A-C), distally opened at the tip of hypopharynx (Fig. 1).

\section{Hypopharinx of Females}

The hypopharynx of female mosquitoes almost invariably are separated from the labrum as a free stylet. Females of

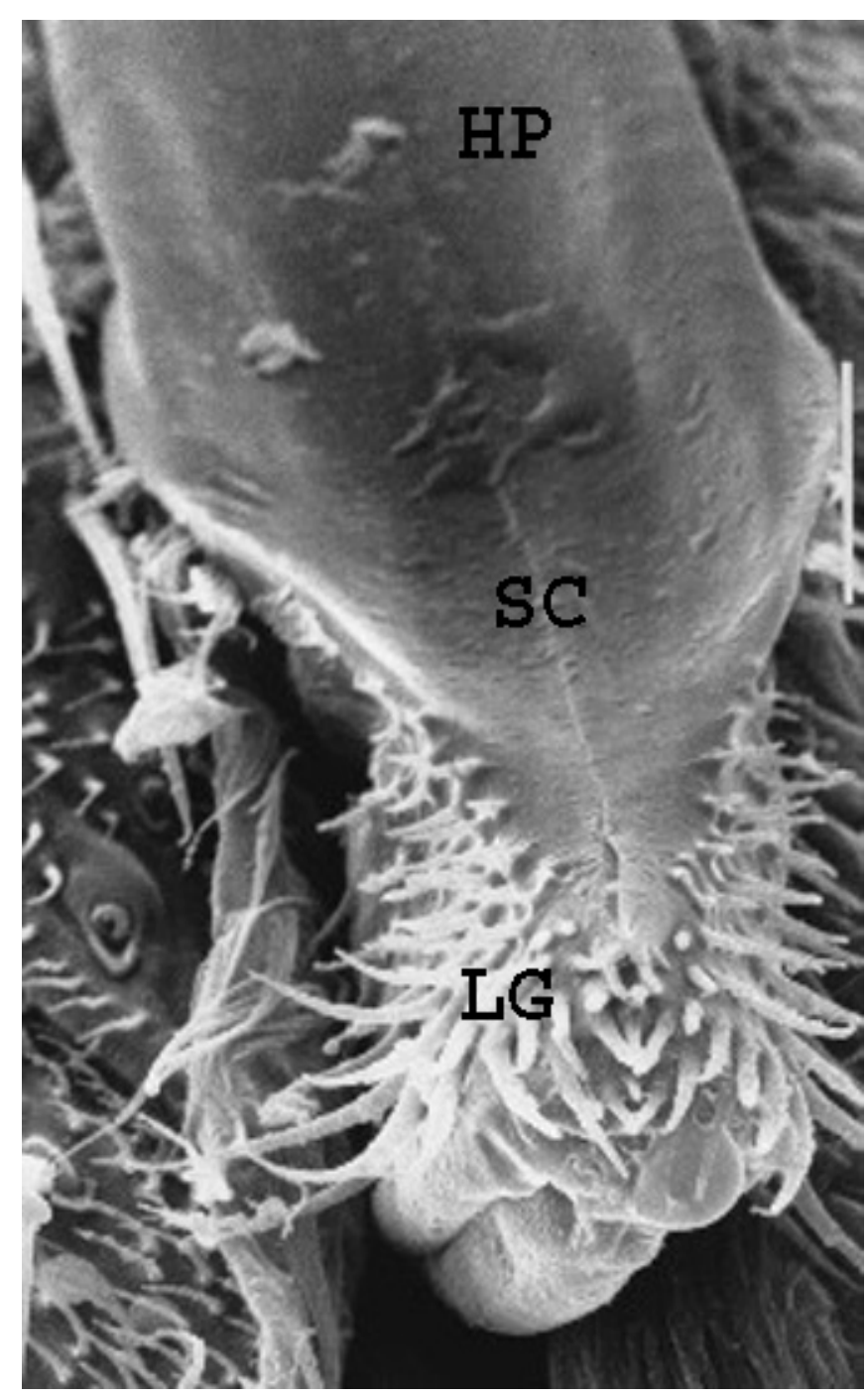

Fig. (1). The hypopharynx tip Ae. aegypti males fused with the ligula. HP, Hypopharynx. SC, Salivary canal. LG, Ligula.

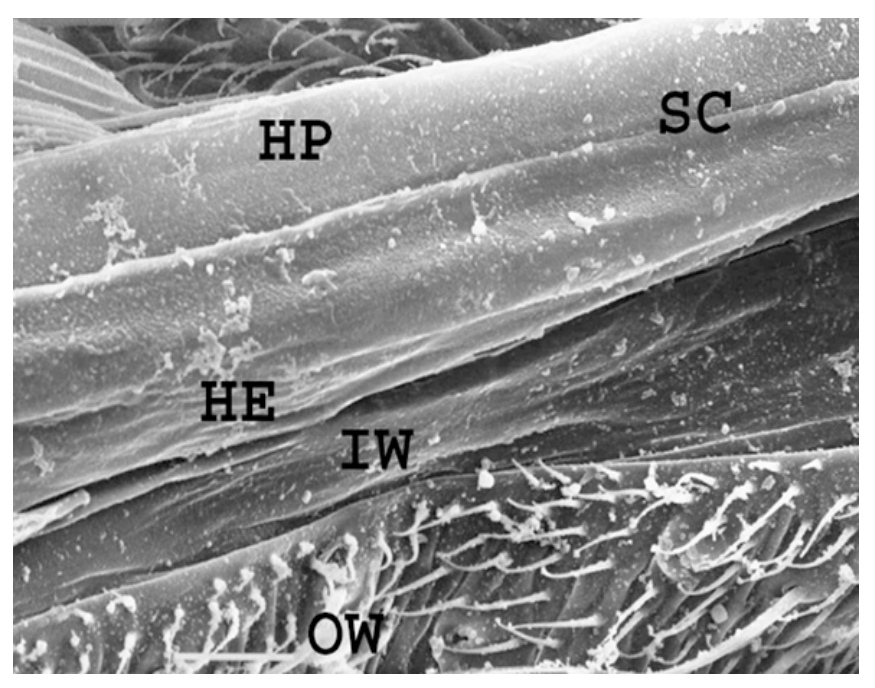

Fig. (2). The hypopharynx trunk of Ae aegypti male inside the labium gutter. HP, Hypopharynx. HE, The hypopharynx edge. SC, Salivary canal. IW, Inner wall of labium. OW, Outer wall of labium. 
anautogenous mosquitoes, represented by the well known domestic mosquitoes Ae. aegypti is a stylet that completely separated from labrum as shown in TEM image (Fig. 5). Mosquitoes that only autogenous at the first batch of their eggs, but ultimately need blood feeding for the rest of their eggs, as predicted, have hypopharynx attributes similar to those of anautogenous one. The females of Culex pipiens molestus Forskal and Ochlerotatus togoi (Theobald), are examples of this group, the partially autogenous species. However, despite the distinct non blood-feeding habits of females Toxorhynchites spp. and Topomyia spp., these lifetime autogenous mosquitoes showing hypopharynx characteristics similar to those of the autogenous females, a free stylet hypopharynx (Fig. 6A,B). The attributes contrasting to those of another lifetime autogenous species, Malaya genurostris, which females have a male like-fused hypopharynx (Fig. 7).

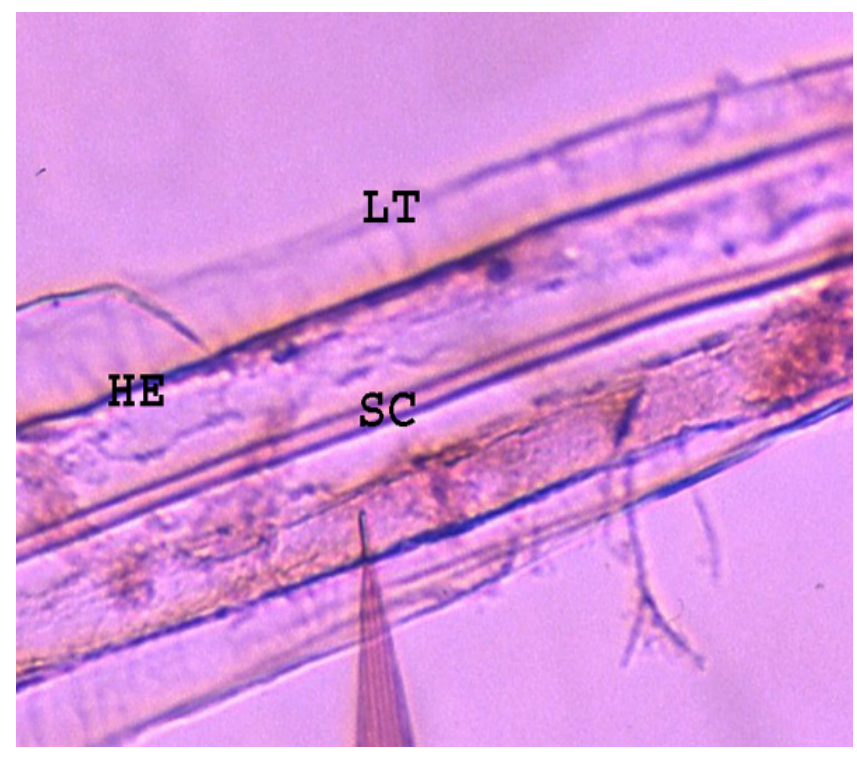

Fig. (3). The detached hypopharynx of a male Ae. aegypti from the labium under a light microscope showing a double edges at each side. The inner edge is the hypopharynx edge (HE), while the outer edge is the attached labium tissue (LT). SC, Salivary canal.
Table 1. Status of Hypopharynx of 44 Species Examined

\begin{tabular}{|c|c|c|}
\hline Species & Female & Male \\
\hline Aedes aegypti (Linneaus) & Free & Fused \\
\hline Aedes albopictus (Skuse) & Free & Fused \\
\hline Aedes flavopictus Yamada & Free & Fused \\
\hline Aedes galloisi Yamada & Free & Fused \\
\hline Aedes paullusi Stone and Farner & Free & Fused \\
\hline Aedes pseudoalbolineatus Brug & Free & Fused \\
\hline Aedes riversi Bohart and Ingram & Free & Fused \\
\hline Aedes scutellaris (Walker) & Free & Fused \\
\hline Anopheles kochi Doenitz & Free & Fused \\
\hline Anopheles lindesayi japonicus Yamada & Free & Fused \\
\hline Anopheles sinensis weidemann & Free & Fused \\
\hline Anopheles stephensi Liston & Free & Fused \\
\hline Anopheles vagus Doenitz & Free & Fused \\
\hline Armigeres sp. 1 & Free & Fused \\
\hline Armigeres sp. 2 & Free & Fused \\
\hline Armigeres sp. 3 & Free & Fused \\
\hline Armigeres subalbatus (Coquillett) & Free & Fused \\
\hline Armigeres theobaldi Barraud & Free & Fused \\
\hline Culex fuscanus weidemann & Free & Fused \\
\hline Culex halifaxii Theobald & Free & Fused \\
\hline Culex kyotoensis Yamaguti and La Casse & Free & Fused \\
\hline Culex pipiens molestus Forskal ${ }^{\mathrm{a}}$ & Free & Fused \\
\hline Culex pipiens pallens Coquillett & Free & Fused \\
\hline Culex quinquefasciatus Say & Free & Fused \\
\hline Culex tritaeniorhyncus Giles & Free & Fused \\
\hline Malaya genurostris Leicester ${ }^{\mathrm{b}}$ & Fused & Fused \\
\hline Mimomyia chamberlaini metallica (Leicester) & Free & Fused \\
\hline
\end{tabular}
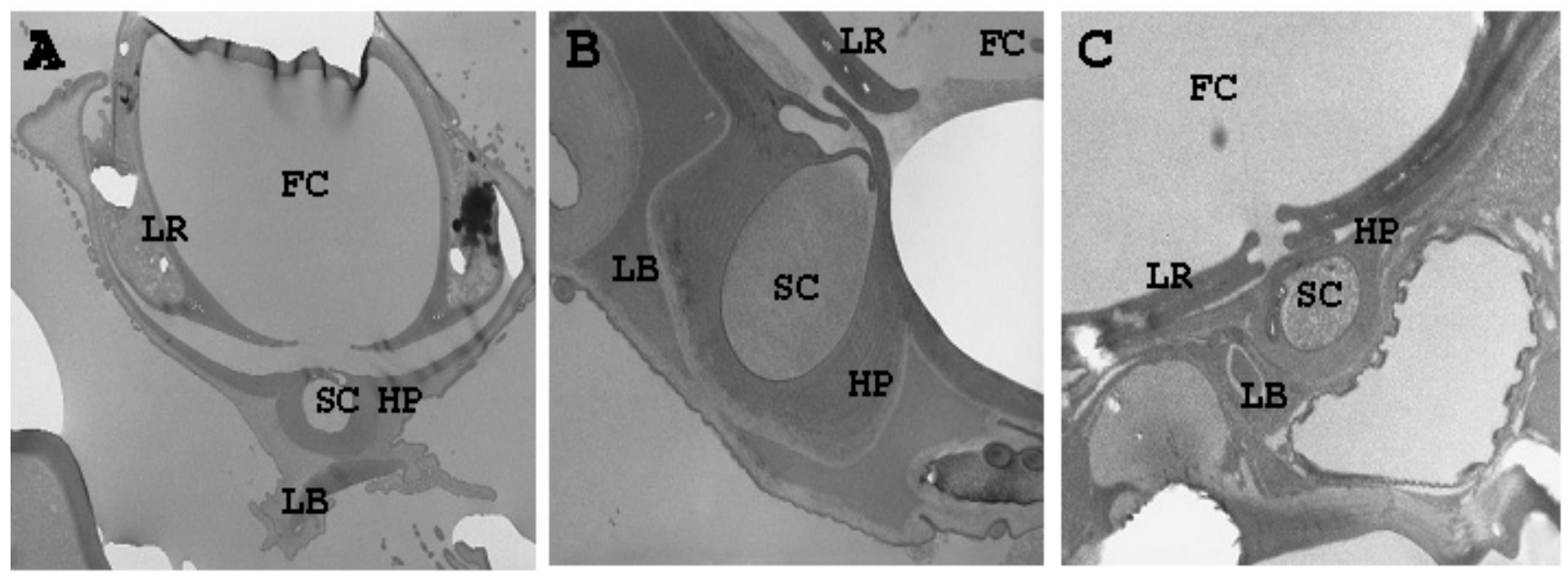

Fig. (4). Transversal sections of the hypopharynx of males of Ae aegypti (A), Tx splendens (B), and To. vijayae (C). FC, Food canal of the labrum. HP, Hypopharynx. LB, Labium. SC, Salivary canal. 
(Table 1) contd.....

\begin{tabular}{|c|c|c|}
\hline Species & Female & Male \\
\hline Ochlerotatus japonicus japonicus (Theobald) & Free & Fused \\
\hline Ochlerotatus poecilius (Theobald) & Free & Fused \\
\hline Ochlerotatus togoi (Theobald) ${ }^{\mathrm{a}}$ & Free & Fused \\
\hline Orthopodomyia anopheloides (Giles) & Free & Fused \\
\hline Orthopodomyia sp. & Free & Fused \\
\hline Topomyia vijayae ramalingan ${ }^{\mathrm{b}}$ & Free & Fused \\
\hline Topomyia yanbarensis ${ }^{\mathrm{b}}$ & Free & Fused \\
\hline Tripteroides aranoides (Theobbald) & Free & Fused \\
\hline Tripteroides bambusa bambusa Yamada & Free & Fused \\
\hline Tripteroides sp. 1 & Free & Fused \\
\hline Tripteroides sp. 2 & Free & Fused \\
\hline Toxorhynchites amboinensis (Doleschall) ${ }^{\mathrm{b}}$ & Free & Fused \\
\hline Toxorhynchites manicatus yaeyama Bohart ${ }^{\mathrm{b}}$ & Free & Fused \\
\hline Toxorhynchites splendens (weidemann) ${ }^{\mathrm{b}}$ & Free & Fused \\
\hline Uranotaenia novobscura novobscura Barraud & Free & Fused \\
\hline Uranotaenia sp. 1 & Free & Fused \\
\hline Uranotaenia sp. 2 & Free & Fused \\
\hline
\end{tabular}

${ }^{a}$ Partially autogenous species; ${ }^{b}$ Lifetime autogenous species.

\section{DISCUSSION}

The present study confirmed the previous reports, that hypopharynx of male mosquitoes fuse with the labium, forms the labio-hypopharynx [4]. Although the tissue of the hypopharynx part in the labio-hypopharynx is clearly different from the tissue of the labium part. Males of all the 44 species representing 12 mosquito genera invariably have a hypopharynx fused with inner wall of the labium, regardless feeding habits of their females. Thus, this attribute was established probably at the earliest stage in mosquito evolution, before extensive diversification.

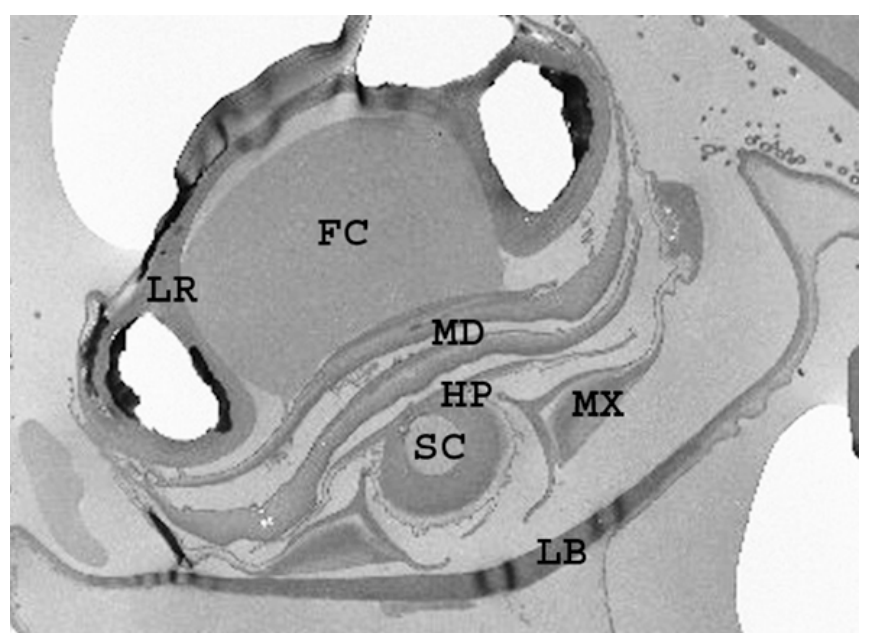

Fig. (5). Transversal section of the proboscis of an Ae. aegypti female. The free hypopharynx (HP) is separated from the labium (LB) by a space accommodating the maxillae (MX). FC, Food canal. LR, Labrum. MD, Mandibles. SC, Salivary canal.

It is interesting to note that males of a sand fly, Lutzomyia migoney Franca (Psychodidae) has a free hypopharynx [7] and males of black flies of the genus Simulium (Simulidae) has a free hypopharynx trunk but its tip fuses with the ligula by a thin transparent membrane (personal observation, unpublished data). Fusion of the hypopharynx and the labium may have taken place after separation of the psychodid line from the lineage including the ancestors of Simulidae and Culicidae.

Despite its fusion with the labium wall, the hypopharynx reaches the tip of the proboscis, with the salivary canal on its
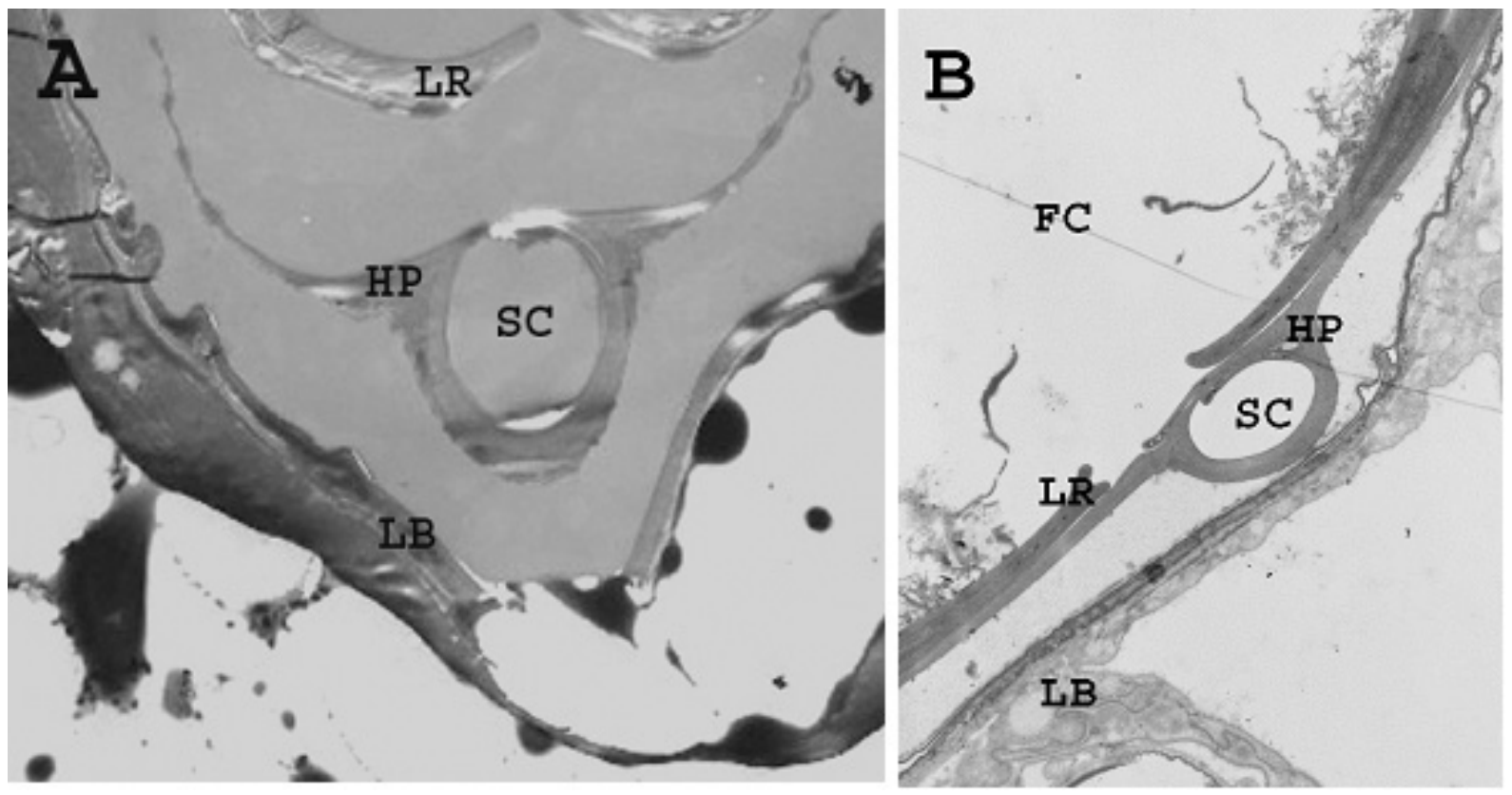

Fig. (6). Transversal sections of the proboscis of autogenous females of Tx. splendens (A), To. vijayae (B) showing the free hypopharynx. FC, Food canal. HP, Hypopharynx. LB, Labium. LR, Labrum. SC, Salivary canal. 
dorsal surface. Differing from male mandibles and maxillae that probably have no function, the salivary canal of males functions as a closed tube by its dorsal overlapping edges, as in females $[1,2,8]$.

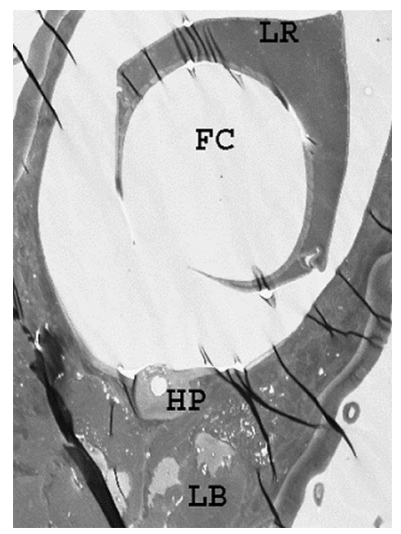

Fig. (7). Transversal section of the proboscis of a Ml. genurostris female showing its unique hypopharynx fused with the labium as in males. FC, Food canal. HP, Hypopharynx. LB, Labium. SC, Salivary canal.

Saliva secreted by salivary glands of males contains $\alpha$ glucosidase, $\alpha$-amylase and bacteriolytic factors, but lack the polypeptide D7, sialokinins I and II, and apyrase which are secreted from the median lobe and the distal portion of lateral lobes of female's salivary glands [2]. The latter three enzymes are responsible for immunoreaction of hosts [9], endothelium-dependent vasodilatation [10] and anti bloodcoagulation [11-13], respectively. Apparently males need saliva for digestion of their main food such as floral and extra-floral nectar and honeydew [14], as well as for protection from pathogenic bacteria in sugar sources [15].

The present study confirmed the previous reports for the free hypopharynx of anautogenous females [4, 5, 8,16-23]. For autogenous females, Hudson reported that an autogenous population of Wyeomyia smithii has a free hypopharynx [20].

We described for the first time, the hypopharynx of females of two partially autogenous (Cx. pipiens molestus and Oc. togoi) and six lifetime autogenous females ( 3 species of Toxorhynchites, 2 Topomyia and 1 Malaya). As in anautogenous mosquitoes, females of life-time autogenous Toxorhynchites and Topomyia, as well as partially autogenous $C x$. pipiens molestus and Oc. togoi have a free hypopharynx. However, females of autogenous Ml. genurostris have shown a fused hypopharynx with the labium wall as the hypopharynx of males.

The unique case of Ml. genurostris, previously known as Harpagomyia [24, 25], may correlate with its remarkable feeding habit among mosquitoes that they are acquiring food from a regurgitation fluid by Crematogaster ants [25-30] with its peculiar proboscis that swollen at the tip [25]. Jacobson $[26,27]$ in Clements [30] described that adults of Malaya splendens feed on a black tree ant, Crematogaster difformis. The adult mosquitoes positioned them self on the ant trails, head uppermost, and rocked back and forth, left and right. Ants ascending the tree walked unhindered between the legs of the mosquitoes, but when an ant descending the trunk reached a mosquito, the mosquito palpated the ant's head with its forelegs and antennae. Usually the ant stopped, pressed its thorax to the tree while raising its abdomen and opening its jaws widely. The mosquito immediately started rocking forward and backward while vibrating its wings. When the ant regurgitated a drop of liquid, the mosquito imbibed it, after which the ant continued on its way.

There are two possibilities of the evolution of hypopharynx of genus Malaya: Firstly, members of the genus retained an old attribute structure of mosquito ancestor's hypopharynx that might be fused before its separation from labium for the purpose of sucking blood. However, this hypothesis is unlikely since a free hypopharynx, as in anautogenous species, is the most common state in female mosquitoes that suggest that their common ancestor had this attribute [31]. More far, the old genera Anopheles that is distributed world wide and placed on the basis of phylogeny tree of mosquitoes based on clasditic analysis [32] has share the free hypopharynx attributes, as well as other autogenous mosquitoes mention above, and hence showing that the hypopharynx of female mosquitoes has a free origin from their common ancestor.

The second hypothesis regarding the attribute of female Malaya hypopharynx is that the fused hypopharynx of this genus might recently derived from a free hypopharynx of its ancestor. This hypothesis is supported at least by three facts: (1) the other life-time autogenous genera (Toxorhynchites and Topomyia) have a free hypopharynx, as well as the partially autogenous species $C x$. pipiens molestus and $O c$. togoi showing that even though these mosquitoes do not use their hypopharynx as blood feeding females did, they still retain the clue of their common ancestor's attributes on hypopharynx and other mouthparts such as maxillas and mandibles in some degrees $[1,20]$, so, the fused hypoharynx of Malaya should derived from an older attribute of their origin; (2) geographic distribution of genus Malaya confined only for Southeast Asia and Papua regions [25,29], showing that this genus might evolved recently in mosquito evolution and hence derived their fused hypopharynx from an older mosquitoes that had a free hypopharynx as other mosquitoes did; (3) phylogeny analyses by Harbach and Kitching, using morphological characters-based cladograms, placed this genus as the most recent group appeared in mosquito evolution [32]. The hypothesis suggests that the genus represents the most advance stage in evolutionary process of the hypopharynx, and that such evolution occurred independently from other autogeneous genera.

Tribes and genera examined by us are still limited in view of the recent classification system of mosquitoes by Harbach and Kitching [32]. Although males of all the species examined have the hypopharynx fused with the labium, there are remain possibilities that different states are discovered for males of the other tribes or genera, especially those put toward the base of the phylogenic tree of mosquitoes based on cladistic analyses of Harbach and Kitching [32]. It is also possible that the hypopharynx fused with the labium is found in life-time autogenous species yet to be examined. We revealed the diverse states of male maxillae and mandibles $[1,6]$, and noted that expansion of the scope to all the mosquito tribes and genera could contribute to phylogenic study of mosquitoes. Though the hypopharynx with functions is much less variable, it could still yield cues to understand the evolution of feeding habits of mosquitoes. 


\section{ACKNOWLEDGEMENTS}

We gratefully thank T. Takahashi, T. Tabata and S. Nakahara for their help in the preparation of electron microscope images, I. Miyagi for the suggestions of staining and dissection methods and providing some samples of Anopheles, Toxorhynchites and Tripteroides, H. S. Yong for his help in mosquito collection in Malaysia, H. Takaoka for his permission to examine some species of black flies (Simulidae) in his laboratory and Y. Eguchi for providing some of Aedes and Culex specimens from laboratory colonies. Mosquito collection in Indonesia was supported by Grant-in-Aid for Monbusho International Scientific Research Program (Field Research) (13576009).

\section{REFERENCES}

[1] Wahid, I., Sunahara, T., Mogi, M. Maxillae and mandibles of male mosquitoes and female autogenous mosquitoes (Diptera: Culicidae). J. Med. Entomol., 2003; 40: 150-159.

[2] Clements, A.N. The Biology of mosquitoes, Vol. 1. Chapman \& Hall, London. 1992. pp. 228-229.

[3] Vizzi, F.F. The mouthparts of the male mosquitoes Anopheles quadrimaculatus Say. Ann. Entomol. Soc. Am., 1953; 46: 496-504.

[4] Snodgrass, R.E. The anatomical life of the mosquitoes. Smiths. Misc. Coll. 139 No. 8. Smithsonian Institution, Washington. 1959.

[5] Christophers, S.R. Aedes aegypti (L.) The Yellow Fever Mosquitoes. Cambridge University Press, Cambridge, UK. 1960.

[6] Wahid, I., Sunahara, T., Mogi, M. Mouthparts of male Aedes (Stegomyia) mosquitoes. J. Am. Mosq. Control Assoc., 2002; 18: 73-80.

[7] Silva, S., Grunewald, J. Comparative study of the mouthparts of males and females of Lutzomyia migonei (Diptera: Psychodidae) by scanning electron microscopy. J. Med. Entomol., 2000; 37: 748753.

[8] Lee, R. Structure and function of the fascicular stylets, and the labral and cibarial sense organs of male and female Aedes aegypti (L.) (Diptera: Culicidae). Quaest entomol., 1974; 10: 187-215.

[9] James, A.A., Blackmer, K., Marinotti, O., Ghosn CR., Racioppi JV. Isolation and characterization of the gene expressing the major salivary gland protein of the female mosquito, Aedes aegypti. Mol. Biochem. Pharmacol., 1991; 44: 245-54.

[10] Ribeiro, J.M.C. Characterization of vasodilator from the salivary glands of the yellow fever mosquito Aedes aegypti. J. Exp. Biol., 1992; 165: 61-71.

[11] Ribeiro, J.M.C., Rossignol, P.A., Spielman, A. Role of mosquito saliva in blood vessel location. J. Exp. Biol., 1984a; 108: 1-7.

[12] Ribeiro, J.M.C., Sarkis, J.J.F., Rossignol, P.A. and Spielman, A. Salivary apyrase of Aedes aegypti: characterization and secretory fate. Comp. Biochem. Physiol., 1984b; 79B: 81-86.

[13] Kroll, M.H., Schafer, A.I. Biochemical mechanisms of platelet activation. Blood, 1989; 74: 1181-1195.
[14] Foster, W.A. Mosquito sugar feeding and reproductive energetics. Annu. Rev. Entomol., 1995; 40: 443-474.

[15] Rossignol, P.A., Lueders, A.M. Bacteriolytic factor in the salivary glands of Aedes aegypti. Comp. Biochem. Physiol. B., 1986; 83 (4): 819-822.

[16] Robinson, G.G. The mouthparts and their function in the female mosquito, Anopheles maculipennis. Parasitology, 1939; 31: 212242.

[17] Walbauer, G.P. The mouthparts of female Psorophora ciliata (Diptera: Culicidae) with the interpretation of the function of the labral muscles J. Morph., 1962; 111:201-215.

[18] Matsuda, R. Morphology and evolution of the insect head. Mem. Am. Entomol. Inst., 1965; 4: 266-282.

[19] Downes, J.A. The ecology of blood-sucking Diptera: an evolutionary perspective, In Ecology and Physiology of Parasites. (Ed.); A.M. Fallis, University of Toronto Press, Toronto. 1971; pp 232238.

[20] Hudson, A. Notes on the piercing mouthparts of three species of mosquitoes (Diptera: Culicidae) viewed with the scanning electron microscope. Can. Entomol., 1970; 102: 501-509.

[21] Larsen, J.R., Owen, W.B. Structure and function of the ligula of the mosquito Culiseta inornata (Williston). Trans. Am. Micros. Soc. 1971; 90: 294-308

[22] Lee, R.M.K.W., Craig, D.A. The labrum and labral sensilla of mosquitoes (Diptera: Culidae): a scanning electron microscope study. Can. J. Zool., 1983; 61: 1568-1579.

[23] Jobling, B. Anatomical drawing of biting flies. British Museum (Natural History), London. 1987.

[24] Edwards, F.W. Diptera fam. Culicidae. In Genera Insectorum (Ed. Wytsman P). Brussel. Fasc. 1932; 194: 258

[25] Bonne-Wepster, J. Synopsis of a hundred common non-anopheline mosquitoes of the greater and lesser Sunda, the Moluccas and New Guinea. Royal Trop. Amsterdam Spec. Pub. No. CXI. Elsevier Publishing Company. Amsterdam. 1954; pp. 20-21.

[26] Jacobson, E. E., in moskito als gast und diebischers schmarotzer der Crematogaster difformis Smith und eine andere schmarotzende fliege. Tjidschrift Entomol., 1909; 52: 158-164.

[27] Jacobson, E. Nahere mitteilungen uber die myrmecophile Culicide Harpagomyia splendens de Meij. Tjidschrift Entomol., 1911; 54: 158-161.

[28] Farquharson, C.O. Harpagomyia and other Diptera fed by Crematogaster ants in S. Nigeria. Proc. Entomol. Soc. Lond., 1918; pp. xxix-xxxix

[29] Miyagi, I. Malaya leei (Wharton) feeding on ants in Papua New Guinea (Diptera: Culicidae). Jap. J. Sanit. Zool., 1981; 32: 332-333.

[30] Clements, A.N. The Biology of mosquitoes, Vol. 2. Chapman \& Hall, London. 1999. pp. 409-410.

[31] Freeman, S., Herron, J.C. Evolutionary Analysis. Pearson Education, Inc. USA. 2004; pp. 57

[32] Harbach, R.E., Kitching, I.J. Phylogeny and classification of the Culicidae (Diptera). Syst. Entomol., 1998; 23: 327-370. 